\title{
Experimental Pharmacotherapy for Dry Eye Disease: A Review
}

This article was published in the following Dove Press journal:

Journal of Experimental Pharmacology

\section{Monica Baiula \\ Santi Spampinato (1D)}

Department of Pharmacy and Biotechnology, University of Bologna, Bologna, Italy
Correspondence: Santi Spampinato

Department of Pharmacy and

Biotechnology, University of Bologna, via

Irnerio 48, Bologna, Italy

$\mathrm{Tel}+39$ 05।209185I

Email santi.spampinato@unibo.it

\begin{abstract}
Dry eye disease (DED) is a complex multifactorial disease showing heterogenous symptoms, including dryness, photophobia, ocular discomfort, irritation and burning but also pain. These symptoms can affect visual function leading to restrictions in daily life activities and reduction in work productivity with a consequently high impact on quality of life. Several pathological mechanisms contribute to the disease: evaporative water loss leads to impairment and loss of tear homeostasis inducing either directly or indirectly to inflammation, in a self-perpetuating vicious cycle. Dysregulated ocular immune responses result in ocular surface damage, which further contributes to DED pathogenesis. Currently, DED treatment is based on a flexible stepwise approach to identify the most beneficial intervention. Although most of the available treatments may control to a certain extent some signs and symptoms of DED, they show significant limitations and do not completely address the needs of patients suffering from DED. This review provides an overview of the emerging experimental therapies for DED. Several promising therapeutic strategies are under development with the aim of dampening inflammation and restoring the homeostasis of the ocular surface microenvironment. Results from early phase clinical trials, testing the effects of EnaC blockers, TRPM8 agonist or mesenchymal stem cells in DED patients, are especially awaited to demonstrate their therapeutic value for the treatment of DED. Moreover, the most advanced experimental strategies in the pipeline for DED, tivanisiran, IL-1R antagonist EBI005 and SkQ1, are being tested in Phase III clinical trials, still ongoing. Nevertheless, although promising results, further studies are still needed to confirm efficacy and safety of the new emerging therapies for DED.
\end{abstract}

Keywords: dry eye disease, inflammation, tivanisiran, IL-1R antagonist, SkQ1

\section{Introduction}

Dry eye disease (DED) is a complex multifactorial disorder associated with a considerable variability in presentation and affecting an increasing number of people worldwide. The definition elaborated by the 2017 International Dry Eye Workshop II (DEWS II) outlines DED as a

Multifactorial disease of the ocular surface characterized by a loss of homeostasis of the tear film, and accompanied by ocular symptoms, in which tear film instability and hyperosmolarity, ocular surface inflammation and damage, and neurosensory abnormalities play etiological roles. ${ }^{1}$

Signs and symptoms of DED are heterogenous: patients can experience persistent irritation, itching or burning, redness, dryness, photophobia, foreign body sensation but also pain. These symptoms can affect visual function and visual performance, 
leading to difficulties and restrictions in performing daily activities such as reading, writing, watching television and driving. In addition, DED can lead to diminished work productivity, decreased general health and well-being, and therefore lead to a reduced quality of life and in some cases to depression. ${ }^{2}$ Moreover, some severe cases, such as those associated with severe Sjögren's syndrome or graft versus host disease (GvHD), can determine real reduced vision due to severe corneal complications.

Etiological classification of DED highlights two main types of the disease: aqueous deficient dry eye (ADDE) and evaporative dry eye (EDE). On the basis of epidemiological and clinical evidences, EDE, mainly caused by meibomian gland dysfunction, is considered the most preponderant form if compared to ADDE, ${ }^{3,4}$ although overlapping forms have been described and as the disease progresses, both evaporative and aqueous deficient components become clinically evident.

The ocular surface (comprising cornea, conjunctiva, limbus, the overlying tear film, eyelids, eyelashes, conjunctival accessory lacrimal glands and goblet cells), the main lacrimal gland, the innervation and meibomian glands (responsible of the production of the outer lipid film, a component of the tear film) compose the lacrimal functional unit. ${ }^{5}$

In physiological conditions, a tight regulation maintains precorneal tear film smooth and optically effective; the loss of this mechanism in DED leads to tear film instability and visual disturbances, ${ }^{6,7}$ thus contributing to ocular surface damage. Several different disorders, including, but not limited to, ocular surface inflammation due to topical preservative toxicity, allergic eye disease, and loss of conjunctival goblet cells or altered mucin expression, due to xerophthalmia, can lead to tear film instability. ${ }^{8}$ Many DED symptoms associate to imbalances of tear film and ocular surface. ${ }^{9}$ Several factors, comprising eyelid and blink abnormalities, deficiencies of tear components and ocular surface, contribute to the loss of tear film homeostasis. ${ }^{10,11}$ These alterations can lead to tear film instability following increased evaporation from the ocular surface; therefore, the aforementioned changes can contribute to the pathogenesis of DED and are considered as triggers of a cycle of events, also referred to as vicious circle $^{8,10-13}$ In addition, oxidative stress also contributes to the pathogenesis of DED. ${ }^{14}$

Irritation, due to environmental or endogenous factors, or microbial stress on the ocular surface activates downstream signaling events that initiate an acute inflammatory response. This leads to the production of proinflammatory cytokines (IL-1 $\alpha$, IL-1 $\beta$, IL-6, TNF- $\alpha$ ), chemokines (CCL3, CCL4) and the production of proteases, such as metalloproteinase-9 (MMP-9) ${ }^{15}$ via MAPK (specifically JNK, ERK and p38) and NF-kB intracellular signaling pathways. ${ }^{16-20}$ As a consequence, inflammatory cells are activated and recruited at the ocular surface and become an additional source of inflammatory mediators. ${ }^{21}$ Inflammatory mediators contribute to a reduced expression of mucins, to apoptotic death of surface epithelial cells ${ }^{22}$ and to loss of goblet cells.

Both innate and adaptive mechanisms are involved in ocular surface immune response. ${ }^{13,23}$ Among the variety of immune cells contributing to the innate immune response, natural killer, dendritic cells (which act as antigen presenting cells, APC), macrophages and $\mathrm{CD} 4^{+} \mathrm{CD}^{+} \mathrm{T}^{-e l l s}{ }^{24-26}$ reside at the ocular surface and can be activated to respond to several stimuli. Proinflammatory cytokines stimulate the maturation of $\mathrm{APC},{ }^{26,27}$ which facilitate the differentiation of several types of $\mathrm{T}$ cells, including memory $\mathrm{T}$ cells, $\mathrm{T}$ helper cells $\left(\mathrm{T}_{\mathrm{H}} 1, \mathrm{~T}_{\mathrm{H}} 2, \mathrm{~T}_{\mathrm{H}} 17\right)^{28}$ and $\mathrm{T}$ regulatory cells, ${ }^{26}$ and their recruitment at the ocular surface. Immunoregulatory processes usually promote the resolution of inflammation but when the homeostatic control mechanisms fail, the immune response is amplified, leading to further inflammation and damage through dysregulated activity of effector T cells and increased production of proinflammatory cytokines. ${ }^{5,29}$ Thus, these effects further stimulate the innate immune response contributing to the vicious circle. ${ }^{21,30}$ In addition, loss of homeostatic mechanisms of the lacrimal functional unit can lead to dysregulation of the innate immune response. $^{31}$

In the last decade, several evidences highlighted the potential role of neurosensory abnormalities in DED pathogenesis: ocular surface sensory neurons can be sensitized in response to physiological stimulation or to inflammatory stimuli (such as inflammation); as a consequence corneal nociceptors can lead to differences in tear flow and blinking, to activation of immune response contributing to neurogenic inflammation. ${ }^{32}$

Several risk factors can lead to a higher predisposition to develop DED, including advancing age, female sex, contact lens use, low humidity environment, and autoimmune disease. ${ }^{7}$ In addition, "environmental" risk factors have been implicated in DED, including topical medications, desiccating conditions, and digital device use. A number of drugs in systemic use, such as antihistamines, beta-blockers, antispasmodics, diuretics and some 
psychotropic drugs, cause a reduction in lacrimal secretion and are risk factors for DED. ${ }^{7,33}$

The multifactorial nature of DED highly complicates the management of this disease; thus, an accurate diagnosis and a proper treatment plan should consider all the underlying etiological mechanisms.

The utmost aim of the DED treatment, through the disruption of the vicious circle, is the restoration of ocular surface homeostasis and the prevention of symptoms exacerbation. Accordingly, a management algorithm has been developed and suggested ${ }^{33}$ as a flexible stepwise approach to identify the most beneficial intervention. Based on disease etiology and severity, DED management can be summarized in four steps. The first step of intervention comprises patient education, dietary modifications, changes in the local environment, identification of other concomitant medication that can be detrimental for DED, usage of ocular lubricants, lid hygiene and warm compresses. ${ }^{33,34}$ If symptoms are not improved with strategies included in step 1, treatments in the second step of DED management include preservative-free artificial tears, punctal plugs, devices to maintain moisture and temperature, intense pulse light therapy. Topical drugs, including corticosteroids, cyclosporine, secretagogues, immunomodulators, antibiotics and lifitegrast are very useful in the treatment of moderate-severe DED. The third step of DED management comprises autologous/allogenic serum eye drops, oral secretagogues and therapeutic contact lenses. The fourth step of DED management is considered when more severe complications are associated with DED symptoms; in these cases chronic corticosteroid therapy, amniotic membrane graft, or surgical intervention can be considered. ${ }^{9,34,35}$

Despite a better understanding of pathogenesis and the availability of multimodal types of treatment, DED remains incompletely treated. Many challenges and limitations in the therapeutic management of the disease are still present. Ocular lubricants represent an important part of DED management. However, they offer only a temporary relief from symptoms and do not influence chronic DED pathogenesis. Moreover, preservative-free lubricant formulations should be preferred to avoid negative effects on the ocular surface following repeated exposure; accordingly, long-term application of artificial tears containing preservatives can lead to modifications in the ocular surface microbiome and epithelial damage. ${ }^{33}$ In addition, dynamic of tear film can be altered by punctal occlusion. Long-term use of topical corticosteroid is not without risk of complications, including ocular hypertension, cataracts and opportunistic infections, ${ }^{33}$ although they are a well-established acute, short-term treatment for DED, especially for recrudescence. Drug formulation for topical ocular administration needs to be improved especially for those hydrophobic drugs, such as cyclosporine. ${ }^{36-38}$

New therapeutic options need to be developed: this review aims to provide an overview of emerging therapies for the treatment of DED that may particularly impact the field in the future. Notably, this review describes innovative approaches for the treatment of DED currently in preclinical and clinical development (Table 1); they are divided on the basis of the mechanism of action, starting from new anti-inflammatory approaches and those combining anti-inflammatory properties with other mechanisms. Finally, emerging therapies acting through different mechanisms of action and cell therapy are described.

\section{Methods}

In this review article, a systematic computerized search of the literature was conducted from inception until November 2020. All English language articles dealing with experimental pharmacotherapy for the treatment of DED were retrieved from the electronic databases PubMed and MEDLINE, then checked for applicability by the authors. The searches were performed by two independent investigators (M.B. and S.S.). The following keywords and MeSH terms were used "dry eye disease," "DED," "experimental treatment," "novel drug," "innovative treatment." All pertinent articles were thoroughly assessed, and their reference lists were searched to identify other potentially relevant studies. The database ClinicalTrials.gov (www.clinicaltrials.gov) was also searched for information about clinical trials.

\section{Novel Emerging Therapies for DED with Anti-Inflammatory Properties Interleukin-I Receptor Antagonists}

Interleukin-1 (IL-1) is a proinflammatory cytokine involved among other inflammatory processes in ocular surface inflammation during DED. ${ }^{39} \mathrm{IL}-1$ receptor antagonists (IL-1Ra), produced by monocytes, macrophages and epithelial cells, competitively blocks the binding of IL-1 to its receptor, suppressing IL-1-mediated pro-inflammatory action. Reduction in IL-1 levels and upregulation of IL1Ra were observed at the ocular surface after treatment with anti-inflammatory drugs, including tetracycline 
Table I Clinical Trials, Aiming to Evaluate the Effects of Novel Emerging Therapies on DED Patients, are Listed Based on the Mechanism of Action

\begin{tabular}{|c|c|c|c|c|c|}
\hline \multirow[t]{2}{*}{ Therapy Name } & \multirow[t]{2}{*}{ Mechanism of Action } & \multicolumn{3}{|c|}{ Clinical Trials } & \multirow[t]{2}{*}{ References } \\
\hline & & $\begin{array}{l}\text { Clinical Trial } \\
\text { Identifier }\end{array}$ & Phase & Ocular Condition or Disease & \\
\hline \multirow[t]{5}{*}{$\begin{array}{l}\text { IL-IR antagonists } \\
\text { (Anakinra, EBI-005) }\end{array}$} & \multirow[t]{5}{*}{ Anti-inflammatory } & NCT0068II09 & $\mathrm{I}-2$ & $\begin{array}{l}\text { DED associated with meibomian gland } \\
\text { dysfunction }\end{array}$ & \multirow[t]{5}{*}{ [43-45] } \\
\hline & & NCT0I 748578 & 1 & DED & \\
\hline & & NCT0I745887 & 1 & DED & \\
\hline & & NCT02405039 & 3 & Moderate to severe DED & \\
\hline & & NCT0I998802 & 3 & Moderate to severe DED & \\
\hline $\begin{array}{l}\text { Serine protease } \\
\text { inhibitor } \\
\text { (UAMC-00050) }\end{array}$ & Anti-inflammatory & - & - & - & [46] \\
\hline $\begin{array}{l}\text { Integrin antagonist } \\
\text { (GW559090) }\end{array}$ & Anti-inflammatory & - & - & - & {$[55,56]$} \\
\hline \multirow{4}{*}{$\begin{array}{l}\text { Resolvin EI analogs } \\
\text { (RX-10045) }\end{array}$} & \multirow[t]{4}{*}{ Promote the resolution phase of inflammation } & NCT0I675570 & 2 & DED & \multirow[t]{4}{*}[57,61-65]{} \\
\hline & & NCT00799552 & 2 & DED & \\
\hline & & NCT0I639846 & 2 & Allergic conjunctivitis & \\
\hline & & NCT02329743 & 2 & $\begin{array}{l}\text { Ocular inflammation and pain in cataract } \\
\text { surgery }\end{array}$ & \\
\hline \multirow[t]{2}{*}{ Lubricin } & \multirow[t]{2}{*}{ Lubricant and anti-inflammatory } & NCT02507934 & $\begin{array}{c}\text { Not } \\
\text { applicable }\end{array}$ & Moderate DED & \multirow[t]{2}{*}{ [68] } \\
\hline & & NCT025I0235 & $\begin{array}{c}\text { Not } \\
\text { applicable }\end{array}$ & Moderate DED & \\
\hline $\begin{array}{l}\text { Artemisinin analog } \\
\text { (SM934) }\end{array}$ & Anti-inflammatory and immunomodulatory & - & - & - & [72] \\
\hline \multirow[t]{3}{*}{ SkQI (Visomitin) } & \multirow[t]{3}{*}{ Antioxidant } & NCT0212130I & 2 & DED & \multirow[t]{3}{*}{ [77] } \\
\hline & & NCT03764735 & 3 & DED & \\
\hline & & NCT04206020 & 3 & DED & \\
\hline Thrombospondin-I & Immunoregulatory & - & - & - & [88] \\
\hline \multirow{2}{*}{$\begin{array}{l}\text { ENaC blockers } \\
(P-32 I)\end{array}$} & \multirow[t]{2}{*}{ Increase ocular hydration } & NCT0283।387 & 2 & Mild to moderate DED & \multirow[t]{2}{*}[94,95]{} \\
\hline & & NCT02824913 & 2 & Tear deficient DED & \\
\hline $\begin{array}{l}\text { TRPM8 agonist } \\
\text { (AR-155I2) }\end{array}$ & Improve tear secretion & NCT04498I82 & 2 & DED & {$[101,102]$} \\
\hline Tivanisiran (SYLI00I) & $\begin{array}{l}\text { Reduce ocular pain/discomfort silencing TRPVI } \\
\text { (by RNA interference) }\end{array}$ & NCT03I08664 & 3 & Moderate to severe DED & {$[106,109]$} \\
\hline \multirow[t]{2}{*}{$\begin{array}{l}\text { Mesenchymal stem } \\
\text { cells }\end{array}$} & \multirow[t]{2}{*}{$\begin{array}{l}\text { Anti-inflammatory, immunomodulatory, restore } \\
\text { goblet cell density and tear production }\end{array}$} & NCT03878628 & $\begin{array}{l}\text { Early } \\
\text { Phase I }\end{array}$ & $\begin{array}{l}\text { Aqueous Deficient DED, } \\
\text { keratoconjunctivitis sicca, aqueous tear } \\
\text { deficiency }\end{array}$ & \multirow[t]{2}{*}[113,114]{} \\
\hline & & NCT046I5455 & 2 & $\begin{array}{l}\text { Keratoconjunctivitis sicca in Sjogren's } \\
\text { syndrome }\end{array}$ & \\
\hline
\end{tabular}


derivatives, topical corticosteroids and cyclosporine A. ${ }^{39}$ Topical administration of IL-1Ra has been successfully used in animal model of ocular diseases. ${ }^{40-42}$ In a mouse model of corneal transplantation IL-1Ra promoted graft survival by suppressing inflammation; ${ }^{40}$ in addition, in a rat model of alkali injury, IL-1Ra significantly decreased corneal inflammation and increased corneal transparency. ${ }^{42}$ In a Phase II randomized clinical trial the effects of the human recombinant IL-1Ra (anakinra) were evaluated in patients having DED associated with meibomian gland dysfunction. ${ }^{43}$ Anakinra was well tolerated and was able to reduce significantly DED symptoms.

EBI-005 is a protein chimera made of IL- $1 \beta$ and anakinra; EBI-005 is able to bind IL-1 receptor with a high affinity abolishing intracellular signaling. ${ }^{44}$ This novel compound has been investigated in vitro and in vivo to unravel its potential as a treatment for ocular surface inflammatory diseases. ${ }^{45}$ Some clinical studies (NCT00681109, NCT01748578, NCT017 45887, NCT02405039, NCT01998802) were performed to evaluate safety and efficacy of EBI-005 in patients with moderate to severe DED, but up to now no results have been released.

\section{Serine Protease Inhibitor}

In several inflammatory conditions, serine proteases play a pivotal role, and therefore represent interesting therapeutic targets for many diseases in which inflammation is an important driver.

In a recent study, the serine protease inhibitor UAMC00050 was evaluated in an in vivo model of DED. ${ }^{46}$ UAMC-00050, a small diphenyl phosphonate with a multitarget inhibition profile, ${ }^{47,48}$ was administered as eye drops in rats subjected to the removal of the exorbital lacrimal gland. UAMC-00050 induced a significant reduction in ocular surface damage, a decrement of IL- $1 \alpha$ and TNF- $\alpha$ levels in tear fluid, although it did not exert any effect on tear volume. The preservation of ocular surface health by UAMC-00050 might correlate with the reduction of serine protease and MMP-9 activity that lead to a less severe inflammatory response. MMP-9 is a critical metalloproteinase produced by corneal epithelial cells in response to hyperosmolar stress, thus reducing its levels reduced corneal extracellular matrix degradation and epithelial cell loss. In addition, UAMC-00050 caused diminished inflammatory cell infiltration in the palpebral conjunctiva; in particular, both $\mathrm{CD}^{+}$and $\mathrm{CD}^{+} 5^{+}$cell were reduced in UAMC-00050 (5 mM)-treated animals in comparison to vehicle-treated and untreated animals. ${ }^{46}$ To date, no clinical trials have been performed to evaluate the effects of UAMC-00050 in individuals with DED.

\section{Integrin Antagonists}

Integrins represent the most important family of cell adhesion receptors and those expressed on leukocytes are deeply involved in the recruitment of immune cells during inflammatory processes. Integrins have already been investigated as interesting therapeutic target for the development of novel drug to treat ocular diseases. ${ }^{49,50}$ Lifitegrast, an $\alpha_{\mathrm{L}} \beta_{2}$ integrin (lymphocyte functionassociated antigen-1, LFA-1) antagonist, was approved in 2016 for the treatment of DED; lifitegrast prevents the self-amplification of the inflammatory immune response involved in DED pathogenesis inducing a significant improvement of signs and symptoms of DED. ${ }^{51,52}$

Similarly, $\alpha_{4} \beta_{1}$ integrin plays a crucial role in inflammation and through the interaction with adhesion molecules, mediates infiltration of neutrophils, eosinophils and T lymphocytes at the conjunctiva. ${ }^{53}$ Accordingly, small molecule $\alpha_{4} \beta_{1}$ integrin antagonists strongly decreased T cell recruitment and infiltration into the ocular surface. ${ }^{54}$

GW559090 is a novel, competitive and high affinity $\alpha_{4}$ integrin antagonist under development as therapeutic strategy for DED. GW559090 was investigated in a murine model of $\mathrm{DED}^{55}$ and in a mouse model of dry eye associated with Sjögren's syndrome. ${ }^{56}$ In both studies, GW559090, blocking $\alpha_{4}$ integrin, significantly led to an improvement of signs and symptoms of DED. Although promising results from preclinical models, GW559090 has not yet been investigated in clinical trials.

\section{Resolvins}

In the past decade a better understanding of inflammatory process has led to the discovery of specialized proresolving mediators that can be considered as new therapeutic approaches for the treatment of ocular surface disease. Resolvins (Rvs) are lipid-derived autacoids playing an important role in the resolution phase of inflammation. ${ }^{57}$

Rvs derive from the metabolism of the polyunsaturated $\omega-3$ fatty acids: resolvins $\mathrm{E}$ (RvEs) are metabolites of eicosapentaenoic acid (EPA), whereas resolvins $\mathrm{D}$ (RvDs) derive from docosahexaenoic acid (DHA). In several in vivo models of inflammation Rvs showed potent anti-inflammatory and proresolution effects. ${ }^{58}$ Moreover, in mouse models of DED, Rvs decreased inflammation and improved tear production. ${ }^{59,60}$ Topical treatment with 
RvE1 induced restoration of epithelial cell density, increased tear production, reduction of infiltrating $\mathrm{CD}^{+}$ T cells and $\mathrm{CD} 11 \mathrm{~b}^{+}$dendritic cells/macrophages. ${ }^{60}$

In a murine model of DED, RvE1 analogs (RX-10001, RX-10005, RX-10008, RX-10065, RX-10045) were able to increase tear production and to inhibit the inflammatory enzyme COX2, therefore strongly contributing to the suppression of inflammation and to the preservation of corneal integrity. ${ }^{57,61-65}$

RX-10045 ([5S,8E,10E,12R]-isopropyl 5,12-hydro xypenta-deca-8,10-dien-6,14-diynoate) is an isopropyl ester synthetic derivative of RvE1. ${ }^{62}$ According to the database ClinicalTrials.gov, two phase II clinical trials (NCT01675570, NCT00799552) were performed to evaluate safety and efficacy of RX-10045 on signs and symptoms of DED in patients but, although both studies are reported to be completed, no results have been published yet. RX-10045 was also investigated in patients for the treatment of allergic conjunctivitis (NCT01639846) and more recently to evaluate efficacy and safety in alleviating ocular inflammation and pain after cataract surgery (NCT02329743). Although RvE1 analogues showed promising results in some preclinical models, to date no further development has been reported, indicating that those compounds are probably no longer in the pipeline for DED.

\section{Omega-3 and Omega-6 Polyunsaturated Fatty Acids}

Based on promising results with resolvin analogues, oral administration of resolvin precursors ( $\omega-3$ and $\omega-6$ fatty acid) has been investigated. Polyunsaturated fatty acid (PUFA) supplements, containing $\omega-3$ and/or $\omega-6$ components, can be derived from plant- and marine-based foods. A recent Cochrane Systematic review assessed the effects of PUFA supplements on signs and symptoms of DED, through the analysis of several clinical trials. ${ }^{66}$ Although the need to improve the consistency of reporting from each trial, a possible role for long-chain $\omega-3$ supplements in managing DED has been reported; the beneficial effects on DED symptoms were observed when $\omega-3$ PUFAs were administered together with standard therapy. ${ }^{66}$

\section{Lubricin}

Lubricin (also named proteoglycan-4) is a mucin-like glycoprotein that is present on the ocular surface and in meibomian glands; ${ }^{67}$ it functions as a lubricant and protects the cornea and conjunctiva against significant shear forces generated during the frequent physiological eyelid blinks, therefore reducing friction between cornea and conjunctiva. These data derive from experiments to evaluate static and kinetic friction at a human corneaeyelid interface. Lubricin was purified from culture media conditioned by articular cartilage disks from mature bovine stifle joints. ${ }^{67}$ Moreover, during inflammation an impaired lubricin biosynthesis has been observed that seems to be involved in ocular surface damage during various types of DED. Accordingly, a reduction of lubricin increases shear stress at the ocular surface, which may lead to inflammation, accumulation of inflammatory mediators (including IL-1 $\alpha$, IL-6, TNF- $\alpha$ ) and proteases (such as MMP-9) in the tear film. ${ }^{67}$ Safety and efficacy of recombinant human lubricin administration were evaluated in a clinical trial in patients with moderate DED. ${ }^{68}$ Recombinant human lubricin was administered locally as eye drops and compared to $0.18 \%$ sodium hyaluronate. Significant improvement in both signs and symptoms of DED was observed after lubricin administration compared to sodium hyaluronate. ${ }^{68}$

\section{Artemisinin Analog}

Another strategy that aims at targeting the inflammatory process involved in the development of DED, is the potential use of artemisinin derivatives. SM934, $\beta$ aminoarteether maleate, is an analog of artemisinin that showed anti-inflammatory and immunomodulatory properties in some inflammatory and autoimmune diseases. ${ }^{69-71}$ The effects of SM934 on DED were investigated in two animal model of DED: scopolamine hydrobromideinduced rodent model, an aqueous-deficient dry eye animal models, and benzalkonium chloride (BAC)-induced rat model, in which BAC induces instability of tear film and excessive evaporation, characteristic features of DED. ${ }^{72}$ Topical instillation of SM934 significantly improved signs of DED, leading to an increased tear secretion and reduced corneal damage; this latter effect could be mediated by a reduction of MMP-9 levels in the corneal epithelium. SM934 significantly reduced the levels of inflammatory mediator (IL-1 $\beta$, TNF- $\alpha$, IL-6, IL-10) and diminished monocyte accumulation in the conjunctival tissues. At molecular levels it has been proposed that SM934 blocks the inflammatory process by downregulating Toll-like receptor 4 (TLR4) and therefore inhibiting the inflammasome activation. ${ }^{72}$ 


\section{Novel Emerging Therapies for DED Acting Through Other Mechanisms of Action \\ SkQI (Visomitin)}

SkQ1 (Visomitin) is a novel synthetic antioxidant that enters the cells and accumulates in the inner leaflet of mitochondrial membrane. It reduces damages induced by overproduction of mitochondrial reactive oxygen species (ROS), through the transition between a reduced and a recharged form. ${ }^{73} \mathrm{SkQ} 1$ has been investigated both in vitro $^{74}$ and in vivo. In a rabbit model of anesthesiainduced DED, SkQ1 prevented pathological changes in the cornea; moreover, it completely abolished clinical signs of DED soon after one day post-anesthesia. ${ }^{75}$ Moreover, SkQ1 reduced TNF $\alpha$ and IL-6 production, ${ }^{75}$ decreased glutathione reductase activity ${ }^{75,76}$ inducing protection of the corneal epithelium. According to the database ClinicalTrials.gov, two clinical trials (NCT03764735, NCT02121301) have been performed and one is ongoing (NCT04206020) to study the effects of SkQ1 in DED patients. On the basis of the first results released, SkQ1 showed to decrease tear film instability and to improve the functional state of the cornea. Through its antioxidant mechanism of action SkQ1 seems to significantly improve symptoms in patients with mild to moderate DED. ${ }^{77}$

\section{Thrombospondin- I}

Thrombospondin-1 (TSP-1) is an immunoregulatory factor generally augmented in inflammatory microenvironment. TSP-1 is produced at high levels by a range of cell types, such as endothelial cells, adipocytes, fibroblasts, smooth muscle cells, monocytes, macrophages. ${ }^{78}$ TSP-1 is transiently released during the acute phase of inflammation. TSP-1 is highly expressed on neutrophils and induces an intense chemotactic response to inflamed tissues. $^{79}$ TSP-1 promotes resolution of inflammation facilitating phagocytosis of damaged cells. ${ }^{80,81}$ Moreover, by binding to CD36, TSP-1 induces macrophage phagocytosis of apoptotic cells. ${ }^{82}$ TSP-1 can also modulate inflammation regulating the functions of dendritic cells; accordingly, high levels of TSP-1 increase the tolerance of dendritic cells to antigens, thereby extinguishing the inflammatory response. ${ }^{81}$ TSP-1 suppresses the ability of APC to sensitize T-cells, thus inhibiting their functions. $^{83}$
At the ocular surface, TSP-1 contributes to maintain immune homeostasis and is produced by corneal epithelial cells, ${ }^{84}$ keratocytes ${ }^{85}$ and corneal endothelial cells. ${ }^{86}$ In chronic form of DED and in Sjögren's syndrome, a TSP1 deficiency has been reported. ${ }^{87}$ In a mouse model of DED, it has been observed that TSP-1 levels were increased in the cornea. ${ }^{88}$ Moreover, recombinant TSP-1, administered topically, decreased expression of proinflammatory cytokines (IL-1 $\beta$, IL-6, IL-23, IL-17A) in the conjunctiva and in the cornea, and ameliorated disease severity. Moreover, topical TSP-1 significantly suppressed corneal dendritic cell maturation. ${ }^{88}$ Within this frame, TSP-1 could represent an interesting innovative approach for DED treatment. Nevertheless, to date, no clinical trials are ongoing to evaluate the effects of TSP-1 for DED treatment.

\section{Epithelial Sodium Channel (EnaC) Blockers}

The ocular surface epithelium controls mucosal hydration coupling $\mathrm{Na}^{+}$absorption and $\mathrm{Cl}^{-}$secretion to create an osmotic gradient: through aquaporin channels water is entrained either decreasing ( $\mathrm{Na}^{+}$absorption) or increasing $\left(\mathrm{Cl}^{-}\right.$secretion) tear volume. ${ }^{89}$ The main channels involved in this process are calcium-activated chloride channel, as the major ocular surface $\mathrm{Cl}^{-}$channel, and epithelial sodium channel (EnaC), responsible for sodium absorption. Targeting EnaC has been proven to increase ocular hydration, suggesting a role for $\mathrm{EnaC}$ in the regulation of tear on the ocular surface. ${ }^{90-93}$ EnaC blockers were investigated to evaluate their potential therapeutic role in DED. ${ }^{94}$ P-301 and P-365, novel EnaC inhibitors, were administered as eye drops in a mouse model of induced aqueous tear deficiency, and an increase in tear production and ocular surface hydration were observed. ${ }^{94} \mathrm{~A}$ small molecule inhibitor of EnaC, P-321, is under development for the treatment of DED; ${ }^{95}$ two phase II randomized, placebo-controlled clinical trials (NCT02831387, NCT02824913 in patients with mild to moderate or tear deficient DED, respectively), aimed at evaluating the effect of $0.017 \%$ P-321 on DED symptoms, have been recently terminated and have shown positive results on all symptoms considered.

\section{TRPM8 Agonists}

The manipulation of the transient receptor potential (TRP) channels on the ocular surface may represent a novel 
option to treat DED. TRP are cation channels associated with chemical irritation and temperature change perception. ${ }^{96}$ Several TRPM8 agonists are under development for different therapeutic purposes; in fact, experimental evidences showed TRPM8 involvement in cold thermal transduction, different life-threatening tumors, and other pathologies, including migraine, urinary tract dysfunction and obesity. ${ }^{97-99}$

TRP channels are expressed in the eye, in particular in the cornea, in the conjunctiva and in the eyelid. TRPM8, a member of the TRP family, is a cold thermoreceptor recently suggested to be involved in DED pathogenesis. ${ }^{32}$ Accordingly, TRPM8 senses changes in osmolarity at the corneal surface and evaporationinduced temperature; ${ }^{32}$ in addition, a reduced tear secretion was observed in TRPM8 knock-out mice. ${ }^{100}$

The TRPM8 agonist Borneol, a compound deriving from traditional Chinese medicine, has been suggested as a potential treatment for DED through increased corneal wetness. $^{101}$

Cryosim-3 (C3, 1-diisopropylphosphorylnonane, CAS Registry Number 1503744-37-8-7), is a novel potent and selective TRPM8 agonist. ${ }^{102}$ The effects of C3 were investigated in vitro and in DED patients; it was administered topically onto the closed eyelids with a cotton wipe soaked with drug solution. C3 was able to significantly improve tear secretion and DED symptoms, without provoking pain or ocular irritation, although the study included young patients with mild to moderated DED; ${ }^{102}$ nonetheless, further studies are necessary to better clarify the efficacy of C3 on signs and symptoms of DED in patient. Recently, a prospective nonrandomized pilot study evaluated the effects of topical $\mathrm{C} 3$ on neuropathic ocular pain in patients with DED. ${ }^{103}$ After one week of treatment C3 improved eye pain intensity, quality of life (driving/watching TV, sleep, enjoying life/relations with other people) and associated factors, including burning sensation, light sensitivity, and tearing. In addition, improved effects on eye pain intensity, quality of life (sleep), and associated factors (burning sensation and light sensitivity) remained improved for one month. ${ }^{103}$

AR-15512 ((1R,2S,5R)-N-(4-methoxyphenyl)-5-methyl2-(1-methylethyl) cyclohexanecarboxamide), formerly known as AVX012 or WS-12, is a potent and selective TRPM8 agonist. ${ }^{104,105}$ A Phase II, multicenter, vehiclecontrolled, double-masked, randomized study (NCT04498182) is ongoing to evaluate efficacy and safety of AR-15512 in subjects with DED.

\section{Tivanisiran}

Tivanisiran, formerly SYL1001, is a 19-base small interfering RNA (siRNA) targeting the human transient receptor potential vanilloid 1 (TRPV1), developed as an ophthalmic treatment for DED. ${ }^{106}$ Tivanisiran acts through RNA interference mechanism, reducing the levels of TRVP1 protein within the cells. TRPV1 is expressed in nociceptive neurons but also in other organs including various eye tissues. ${ }^{107}$ TRPV1 is a cation channel that acts as a nociceptive transducer. Several stimuli, such as hyperosmolarity and inflammatory mediators, can activate TRPV1 leading to pain signal that are transmitted to the brain. Tivanisiran has been investigated both in vitro and in vivo using models to study DED, showing promising results. In different human and rabbit cell lines tivanisiran selectively silenced TRPV1 without affecting other receptors, with the exception of TRPV4. ${ }^{106}$ In a capsaicininduced ocular pain model in New Zealand white rabbis, tivanisiran showed analgesic effect. ${ }^{108}$ In clinical trials tivanisiran, administered as preservative-free eye drops, was well tolerated and able to reduce ocular pain/discomfort in patients with moderate to severe DED. ${ }^{109}$ Additional results on tivanisiran efficacy will be obtained from a double-masked Phase 3 study (NCT03108664) in patients with moderate to severe DED, still ongoing.

\section{Stem Cell Therapy for DED Mesenchymal Stem Cells}

Several blood derivatives can be used for the treatment of various ocular diseases including DED. ${ }^{110}$ Blood sources can derive from autologous serum, allogenic peripheral blood and umbilical cord blood (UCB). Serum-based eye drops contain high concentration of growth factors, cell adhesion molecules and biochemical components that make them more similar to natural tears; they perform lubricating, antimicrobial, and anti-inflammatory functions. ${ }^{111}$ DED patients treated with serum-based eye drops experienced a fast epithelial healing rate, increased goblet cell density and a significant improvement in symptoms. $^{110}$

$\mathrm{UCB}$, whose main application is hematological stem cells transplantation for the treatment of hematological diseases, can also be used as a source of other cells, including mesenchymal stem cells (MSCs) for replacement therapy. ${ }^{110}$

MSCs are multipotent stem cells able to differentiate into several cell types, ${ }^{112}$ and possess immunomodulatory 
properties. In a rabbit model of Sjögren's syndrome dry eye, a chronic autoimmune disorders that affects lacrimal glands and ocular surface among other tissues, human umbilical cord MSCs (hUC-MSCs) were intravenously infused after disease onset. ${ }^{113}$ Administration of hUCMSCs induced a significant improvement of the clinical symptoms, including tear production and conjunctival impairment. The proposed mechanism by which hUCMSCs are effective in the relief of DED symptoms seems mediated by suppression of the inflammatory process. Accordingly, animal treated with hUC-MSCs showed a reduction of proinflammatory cytokine production (TNF$\alpha$ and IL-6) and an increment of anti-inflammatory cytokines (IL-10, TGF- $\beta$ ). Moreover, the therapeutic effects of hUC-MSCs can be partly due to their modulation of macrophage polarization: hUC-MSCs treatment promoted anti-inflammatory M2 macrophage polarization in lacrimal glands, probably contributing to suppress the inflammatory response and to enhance reparative activities. ${ }^{113}$

In a mouse model of inflammation-induced DED, human or mouse MSCs, administered by periorbital infusion, were able to suppress inflammation protecting the ocular surface; in addition, MSCs induced restoration of tear production and conjunctival goblet cells. ${ }^{114}$

The potential beneficial effects of MSCs were investigated in patients with dry eye associated with chronic GVHD (cGVHD). ${ }^{115}$ cGVHD arises as a serious longterm complication of allogenic bone marrow transplantation and can frequently cause ocular surface damage, ${ }^{116}$ associated to inflammatory and fibrotic processes. MSCs were administered to patients with refractory DED secondary to cGVHD by intravenous injection; a clinical improvement was observed in 12 out of 22 patients. In addition, MSCs triggered the generation of $\mathrm{CD} 8^{+} \mathrm{CD} 28^{-}$ $\mathrm{T}$ cells, a subset of $\mathrm{CD}^{+} \mathrm{T}$ cells which can inhibit the lymphocyte response and prevent the activation and effector function of autoreactive T cells. Consequently, it was proposed that MSCs likely control the Th1/Th2 homeostasis, which is dysregulated in cGVHD, by generating $\mathrm{CD} 8^{+} \mathrm{CD} 28^{-}$T cells. ${ }^{115}$

These results demonstrate that MSCs represent a safe and potentially effective approach for the treatment of DED patients and other inflammatory ocular diseases. Accordingly, two clinical trials (NCT03878628, NCT04615455) are ongoing to ascertain efficacy and safety of MSCs in patients with aqueous deficient DED and DED associated to Sjögren's Syndrome, respectively.

\section{Conclusion}

Despite several therapeutic strategies available, currently there is no cure for DED that represents a complex condition whose complete pathogenesis is not entirely uncovered. Nevertheless, the increasing prevalence and demands from patients have accelerated the development of novel drugs. Therapeutic agents for DED offer a provisional and limited control of signs and symptoms, mainly based on increased lubrification and reducing inflammation. In many cases, the available treatments offer a good control of the disease, but the scarcity of long-term marketed drug represents a relevant problem. The only drug approved for long-term use is cyclosporine, and lifitegrast has been approved only in one country.

This review summarizes innovative approaches for the treatment of DED currently in preclinical and clinical development, divided on the basis of their mechanism of action. Some innovative approaches (including serine protease inhibitor, integrin antagonist, artemisinin analog, and TSP-1) have not yet been tested in humans; nevertheless, clinical trials testing these experimental treatments are awaited to verify their efficacy in patients suffering from DED. Moreover, several experimental strategies under development for DED (such as IL-1R antagonists, serine protease inhibitor, integrin antagonist, resolvin analogs, and $\omega-3$ and $\omega-6$ PUFAs) target the inflammatory response involved in its pathogenesis; these innovative strategies should prove to be more effective than anti-inflammatory drugs already approved for DED therapy.

Phase I and II clinical trials represent an early evaluation of efficacy and safety of emerging drugs in patients. Therefore, results from early phase clinical trials are particularly crucial as they will authorize or discontinue the following step of clinical evaluation for a developing novel drug. Results from ongoing clinical trials on EnaC blockers, TRPM8 agonist and MSCs are especially awaited to confirm their therapeutic value for the treatment of DED.

The most advanced experimental strategies in the pipeline for DED are tivanisiran, IL-1R antagonist EBI-005 and SkQ1 that are being tested in Phase III clinical trials, still ongoing.

Looking at inflammation from a different point of view, resolvins could be promising agents for DED. Considering that pro-resolving molecules lead to successful resolution of inflammation and after that rapid reestablishment of tissue homeostasis, drugs able to 
rebalance the equilibrium toward homeostasis could be useful to bring to an end acute and chronic inflammatory response. Resolvin analogs have been under development since 2008 but although promising results in preclinical models and in some clinical trials already concluded, those pro-resolving compounds are probably no longer in the pipeline for DED treatment.

Notably, MSCs implantation by different routes has raised very interesting expectations in the treatment of DED thanks to their immunomodulatory and regenerative properties; data on safety and efficacy of MSCs in DED patients are particularly awaited to confirm MSCs as a new perspective in the management of this disease.

In conclusion, several promising new therapeutic strategies are under development, but further studies are necessary to demonstrate their value, efficacy, and safety in the treatment of DED.

DED is a multifactorial disease and the development of novel therapeutic strategies that would target more comprehensively different aspects of the pathogenesis would offer new opportunities to provide a better control of signs and symptoms of DED regardless the underlying etiology.

\section{Abbreviations}

DED, dry eye disease; DEWS II, 2017 International Dry Eye Workshop II; GvHD, graft versus host disease; ADDE, aqueous deficient dry eye; EDE, and evaporative dry eye; IL-1, interleukin-1; IL-6, interleukin-6; TNF- $\alpha$, tumor necrosis factor- $\alpha$; CCL3, C-C motif ligand 3; CCL4, C-C motif ligand 4; MMP-9, metalloprotease-9; JNK, c-JUN N-terminal kinase; MAPK, mitogen activated protein kinase; ERK, extracellular signal-regulated kinases; NFkB, nuclear factor kappa-lightchain-enhancer of activated B cells; APC, antigen presenting cells; IL-1Ra, interleukin-1 receptor antagonist; LFA-1, lymphocyte function-associated antigen-1; Rvs, resolvins; RvEs, resolvins E; EPA, eicosapentaenoic acid; DHA, docosahexaenoic acid; RvDs, resolvins D; ROS, reactive oxygen species; PUFA, polyunsaturated fatty acid; BAC, benzalkonium chloride; TLR4, toll-like receptor 4; IL-10, interleukin-10; TSP-1, thrombospondin-1; IL-23, interleukin-23; IL-17A, interleukin-17A; UCB, umbilical cord blood; MSC, mesenchymal stem cell; hUC-MSCs, human umbilical cord MSCs; TGF- $\beta$, transforming growth factor- $\beta$; cGVHD, chronic graft-versushost disease; ENaC, epithelial sodium channel; TRP, transient receptor potential; TRPM8, Transient receptor potential cation channel subfamily M (melastatin) member 8; C3, cryosim-3; TRPV1, transient receptor potential vanilloid 1; siRNA, small interfering RNA.

\section{Author Contributions}

All authors made a significant contribution to the work reported, whether that is in the conception, study design, execution, acquisition of data, analysis and interpretation, or in all these areas; took part in drafting, revising or critically reviewing the article; gave final approval of the version to be published; have agreed on the journal to which the article has been submitted; and agree to be accountable for all aspects of the work.

\section{Funding}

This work was supported by grants from the University of Bologna RFO 2018, RFO 2019, RFO 2020 to MB and SS; and from a research grant from "Fondazione Cassa di Risparmio in Bologna" (2018/0347).

\section{Disclosure}

The authors declare that the research was conducted in the absence of any commercial or financial relationships that could be construed as a potential conflict of interest.

\section{References}

1. Craig JP, Nichols KK, Akpek EK, et al. TFOS DEWS II definition and classification report. Ocul Surf. 2017;15(3):276-283. doi:10.1016/j.jtos.2017.05.008

2. Stapleton F, Alves M, Bunya VY, et al. TFOS DEWS II epidemiology report. Ocul Surf. 2017;15(3):334-365. doi:10.1016/j. jtos.2017.05.003

3. Shimazaki J, Goto E, Ono M, Shimmura S, Tsubota K. Meibomian gland dysfunction in patients with Sjogren syndrome. Ophthalmology. 1998;105(8):1485-1488. doi:10.1 016/S0161-6420(98)98033-2

4. Lemp MA, Crews LA, Bron AJ, Foulks GN, Sullivan BD. Distribution of aqueous-deficient and evaporative dry eye in a clinic-based patient cohort: a retrospective study. Cornea. 2012;31(5):472-478. doi:10.1097/ICO.0b013e318225415a

5. Stern ME, Schaumburg CS, Pflugfelder SC. Dry eye as a mucosal autoimmune disease. Int Rev Immunol. 2013;32(1):19-41. doi:10.3109/08830185.2012.748052

6. Rieger G. The importance of the precorneal tear film for the quality of optical imaging. $\mathrm{Br} J$ Ophthalmol. 1992;76 (3):157-158. doi:10.1136/bjo.76.3.157

7. Bron AJ, Tomlinson A, Foulks GN, et al. Rethinking dry eye disease: a perspective on clinical implications. Ocul Surf. 2014;12 (2 SUPPL.). doi:10.1016/j.jtos.2014.02.002

8. Baudouin C, Messmer EM, Aragona P, et al. Revisiting the vicious circle of dry eye disease: a focus on the pathophysiology of meibomian gland dysfunction. Br J Ophthalmol. 2016;100 (3):300-306. doi:10.1136/bjophthalmol-2015-307415

9. Zhang X, Vimalin Jeyalatha M, Qu Y, et al. Dry eye management: targeting the ocular surface microenvironment. Int $J \mathrm{Mol} \mathrm{Sci}$. 2017;18(7):1398. doi:10.3390/ijms 18071398

10. Willcox MDP, Argüeso P, Georgiev GA, et al. TFOS DEWS II tear film report. Ocul Surf. 2017;15(3):366-403. doi:10.1016/j. jtos.2017.03.006 
11. Bron AJ, de Paiva CS, Chauhan SK, et al. TFOS DEWS II pathophysiology report. Ocul Surf. 2017;15(3):438-510. doi:10.1016/j.jtos.2017.05.011

12. Lemp MA, Baudouin C, Baum J, et al. The definition and classification of dry eye disease: report of the definition and classification subcommittee of the international dry eye workshop (2007). Ocul Surf. 2007;5:75-92. Ethis Communications, Inc. doi:10.1016/s1542-0124(12)70081-2

13. Periman LM, Perez VL, Saban DR, Lin MC, Neri P. The immunological basis of dry eye disease and current topical treatment options. J Ocul Pharmacol Ther. 2020;36(3):137-146. doi:10.1089/jop.2019.0060

14. Heidari M, Noorizadeh F, Wu K, Inomata T, Mashaghi A. Dry eye disease: emerging approaches to disease analysis and therapy J Clin Med. 2019;8(9):1439. doi:10.3390/jcm8091439

15. De Paiva CS, Corrales RM, Villarreal AL, et al. Corticosteroid and doxycycline suppress MMP-9 and inflammatory cytokine expression, MAPK activation in the corneal epithelium in experimental dry eye. Exp Eye Res. 2006;83(3):526-535. doi:10.1016/j. exer.2006.02.004

16. Li DQ, Chen Z, Song XJ, Luo L, Pflugfelder SC. Stimulation of matrix metalloproteinases by hyperosmolarity via a JNK pathway in human corneal epithelial cells. Invest Ophthalmol Vis Sci. 2004;45(12):4302-4311. doi:10.1167/iovs.04-0299

17. Li DQ, Luo L, Chen Z, Kim HS, Song XJ, Pflugfelder SC. JNK and ERK MAP kinases mediate induction of IL- $1 \beta$, TNF- $\alpha$ and IL-8 following hyperosmolar stress in human limbal epithelial cells. Exp Eye Res. 2006;82(4):588-596. doi:10.1016/j.exer.2005.08.019

18. Luo L, Li DQ, Corrales RM, Pflugfelder SC. Hyperosmolar saline is a proinflammatory stress on the mouse ocular surface. Eye Contact Lens. 2005;31(5):186-193. doi:10.1097/01.ICL.00001 62759.79740 .46

19. Lee JH, Kim M, Im YS, Choi W, Byeon SH, Lee HK. NFAT5 induction and its role in hyperosmolar stressed human limbal epithelial cells. Invest Ophthalmol Vis Sci. 2008;49 (5):1827-1835. doi:10.1167/iovs.07-1142

20. Luo L, Li DQ, Doshi A, Farley W, Corrales RM, Pflugfelder SC. Experimental dry eye stimulates production of inflammatory cytokines and MMP-9 and activates MAPK signaling pathways on the ocular surface. Invest Ophthalmol Vis Sci. 2004;45 (12):4293-4301. doi:10.1167/iovs.03-1145

21. Baudouin C. The pathology of dry eye. Surv Ophthalmol. 2001;45(SUPPL. 2):S211-S220. doi:10.1016/S0039-6257(00) 00200-9

22. Yeh S, Song XJ, Farley W, Li DQ, Stern ME, Pflugfelder SC. Apoptosis of ocular surface cells in experimentally induced dry eye. Invest Ophthalmol Vis Sci. 2003;44(1):124-129. doi:10.1167/ iovs.02-0581

23. Schaumburg CS, Siemasko KF, De Paiva CS, et al. Ocular surface APCs are necessary for autoreactive T cell-mediated experimental autoimmune lacrimal keratoconjunctivitis. J Immunol. 2011;187 (7):3653-3662. doi:10.4049/jimmunol.1101442

24. Zhang X, Volpe EA, Gandhi NB, et al. NK cells promote Th-17 mediated corneal barrier disruption in dry eye. PLoS One. 2012;7 (5). doi:10.1371/journal.pone.0036822

25. Strauss-Albee DM, Horowitz A, Parham P, Blish CA. Coordinated regulation of $\mathrm{NK}$ receptor expression in the maturing human immune system. J Immunol. 2014;193(10):4871-4879. doi:10.4049/jimmunol.1401821

26. Stevenson W, Chauhan SK, Dana R. Dry eye disease: an immune-mediated ocular surface disorder. Arch Ophthalmol. 2012;130(1):90-100. doi:10.1001/archophthalmol.2011.364

27. Hamrah P, Liu Y, Zhang Q, Dana MR. Alterations in corneal stromal dendritic cell phenotype and distribution in inflammation. Arch Ophthalmol. 2003;121(8):1132-1140. doi:10.1001/archoph t.121.8.1132
28. El Annan J, Chauhan SK, Ecoiffier T, Zhang Q, Saban DR, Dana R. Characterization of effector T cells in dry eye disease. Invest Ophthalmol Vis Sci. 2009;50(8):3802-3807. doi:10.1167/ iovs.08-2417

29. Stern ME, Schaumburg CS, Dana R, Calonge M, Niederkorn JY, Pflugfelder SC. Autoimmunity at the ocular surface: pathogenesis and regulation. Mucosal Immunol. 2010;3(5):425-442. doi:10.1038/mi.2010.26

30. Bron AJ, Yokoi N, Gaffney E, Tiffany JM. Predicted phenotypes of dry eye: proposed consequences of its natural history. Ocul Surf. 2009;7(2):78-92. doi:10.1016/S1542-0124(12)70299-9

31. Barabino S, Chen Y, Chauhan S, Dana R. Ocular surface immunity: homeostatic mechanisms and their disruption in dry eye disease. Prog Retin Eye Res. 2012;31(3):271-285. doi:10.1016/j. preteyeres.2012.02.003

32. Belmonte C, Nichols JJ, Cox SM, et al. TFOS DEWS II pain and sensation report. Ocul Surf. 2017;15(3):404-437. doi:10.1016/j. jtos.2017.05.002

33. Jones L, Downie LE, Korb D, et al. TFOS DEWS II management and therapy report. Ocul Surf. 2017;15(3):575-628. doi:10.1016/j. jtos.2017.05.006

34. Şimşek C, Doğru M, Kojima T, Tsubota K. Current management and treatment of dry eye disease. Turk J Ophthalmol. 2018;48 (6):309-313. doi:10.4274/tjo.69320

35. Rouen PA, White ML. Dry eye disease: prevalence, assessment, and management. Home Healthc. 2018;36(2):74-83. doi:10.1097/ NHH.0000000000000652

36. Lallemand F, Schmitt M, Bourges JL, Gurny R, Benita S, Garrigue JS. Cyclosporine A delivery to the eye: a comprehensive review of academic and industrial efforts. Eur J Pharm Biopharm. 2017;117:14-28. doi:10.1016/j.ejpb.2017.03.006

37. Agarwal P, Craig JP, Rupenthal ID. Formulation considerations for the management of dry eye disease. Pharmaceutics. 2021;13 (2):207. doi:10.3390/pharmaceutics 13020207

38. Periman LM, Mah FS, Karpecki PM. A review of the mechanism of action of cyclosporine A: the role of cyclosporine a in dry eye disease and recent formulation developments. Clin Ophthalmol. 2020;14:4187-4200. doi:10.2147/OPTH.S279051

39. Solomon A, Dursun D, Liu Z, Xie Y, Macri A, Pflugfelder S. Proand anti-inflammatory forms of interleukin-1 in the tear fluid and conjunctiva of patients with dry-eye disease - PubMed. Invest Ophthalmol Vis Sci. 2001;42(10):2283-2292.

40. Yamada J, Zhu S-N, Streilein W, Dana R. Interleukin-1 receptor antagonist therapy and induction of anterior chamber-associated immune deviation-type tolerance after corneal transplantation. Invest Ophthalmol Vis Sci. 2000;41:4203-4208.

41. Keane-Myers A, Miyazaki D, Dekaris I, Ono S, Dana M. Prevention of allergic eye disease by treatment with IL-1 receptor antagonist - PubMed. Invest Ophthalmol Vis Sci. 1999;40 (12):3014-3016.

42. Yamada J, Dana MR, Sotozono C, Kinoshita S. Local suppression of IL-1 by receptor antagonist in the rat model of corneal alkali injury. Exp Eye Res. 2003;76(2):161-167. doi:10.1016/S00144835(02)00293-2

43. Amparo F, Dastjerdi MH, Okanobo A, et al. Topical interleukin 1 receptor antagonist for treatment of dry eye disease: a randomized clinical trial. JAMA Ophthalmol. 2013;131(6):715-723. doi:10.1001/jamaophthalmol.2013.195

44. Hou J, Townson SA, Kovalchin JT, et al. Design of a superior cytokine antagonist for topical ophthalmic use. Proc Natl Acad Sci $\quad U \quad S \quad$ A. 2013;110(10):3913-3918. doi:10.1073/pnas.1 217996110

45. Kovalchin J, King B, Masci A, et al. Preclinical development of EBI-005: an IL-1 receptor-1 inhibitor for the topical ocular treatment of ocular surface inflammatory diseases. Eye Contact Lens. 2018;44(3):170-181. doi:10.1097/ICL.0000000000000414 
46. Joossen C, Baán A, Moreno-Cinos C, et al. A novel serine protease inhibitor as potential treatment for dry eye syndrome and ocular inflammation. Sci Rep. 2020;10(1). doi:10.1038/ s41598-020-74159-w

47. Joossens J, Ali OM, El-Sayed I, et al. Small, potent, and selective diaryl phosphonate inhibitors for urokinase-type plasminogen activator with in vivo antimetastatic properties. $J$ Med Chem. 2007;50(26):6638-6646. doi:10.1021/jm700962j

48. Ceuleers H, Hanning N, Heirbaut J, et al. Newly developed serine protease inhibitors decrease visceral hypersensitivity in a post-inflammatory rat model for irritable bowel syndrome. $\mathrm{Br}$ J Pharmacol. 2018;175(17):3516-3533. doi:10.1111/bph.14396

49. Dattoli SD, Baiula M, De Marco R, et al. DS-70, a novel and potent $\alpha 4$ integrin antagonist, is an effective treatment for experimental allergic conjunctivitis in guinea pigs. $\mathrm{Br} J$ Pharmacol. 2018;175(20):3891-3910. doi:10.1111/bph.14458

50. Qasem AR, Bucolo C, Baiula M, et al. Contribution of $\alpha 4 \beta 1$ integrin to the antiallergic effect of levocabastine. Biochem Pharmacol. 2008;76(6):751-762. doi:10.1016/j.bcp.2008.07.007

51. Haber SL, Benson V, Buckway CJ, Gonzales JM, Romanet D, Scholes B. Lifitegrast: a novel drug for patients with dry eye disease. Ther Adv Ophthalmol. 2019;11:2515841419870366. doi:10.1177/2515841419870366

52. Donnenfeld ED, Perry HD, Nattis AS, Rosenberg ED. Lifitegrast for the treatment of dry eye disease in adults. Expert Opin Pharmacother. 2017;18(14):1517-1524. doi:10.1080/ 14656566.2017.1372748

53. Baiula M, Spampinato S, Gentilucci L, Tolomelli A. Novel ligands targeting $\alpha 4 \beta 1$ integrin: therapeutic applications and perspectives. Front Chem. 2019;7:489. doi:10.3389/fchem.20 19.00489

54. Ecoiffier T, El Annan J, Rashid S, Schaumberg D, Dana R. Modulation of integrin $\alpha 4 \beta 1$ (VLA-4) in dry eye disease. Arch Ophthalmol. 2008;126(12):1695-1699. doi:10.1001/archopht.126.12.1695

55. Krauss AH, Corrales RM, Pelegrino FSA, Tukler-Henriksson J, Pflugfelder SC, de Paiva CS. Improvement of outcome measures of dry eye by a novel integrin antagonist in the murine desiccating stress model. Invest Ophthalmol Vis Sci. 2015;56(10):5888-5895. doi:10.1167/iovs.15-17249

56. Contreras-Ruiz L, Mir FA, Turpie B, Krauss AH, Masli S. Sjögren's syndrome associated dry eye in a mouse model is ameliorated by topical application of integrin $\alpha 4$ antagonist GW559090. Exp Eye Res. 2016;143:1-8. doi:10.1016/j. exer.2015.10.008

57. Hesselink JMK, Chiosi F, Costagliola C. Resolvins and aliamides: lipid autacoids in ophthalmology - what promise do they hold? Drug Des Devel Ther. 2016;10:3133-3141. doi:10.2147/DDDT. S112389

58. Ji RR, Xu ZZ, Strichartz G, Serhan CN. Emerging roles of resolvins in the resolution of inflammation and pain. Trends Neurosci. 2011;34(11):599-609. doi:10.1016/j.tins.2011.08.005

59. Dartt DA, Hodges RR, Li D, Shatos MA, Lashkari K, Serhan CN. Conjunctival goblet cell secretion stimulated by leukotrienes is reduced by resolvins D1 and E1 to promote resolution of inflammation. J Immunol. 2011;186(7):4455-4466. doi:10.4049/ jimmunol.1000833

60. Li N, He J, Schwartz CE, Gjorstrup P, Bazan HEP. Resolvin E1 improves tear production and decreases inflammation in a dry eye mouse model. J Ocul Pharmacol Ther. 2010;26(5):431-439. doi:10.1089/jop.2010.0019

61. Schwartz CE, Savinainen A, Gjorstrup P. Resolvin analogs with pharmacokinetic properties suitable for topical administration to treat ocular diseases. Invest Ophthalmol Vis Sci. 2008;49(5):3179.

62. Cholkar K, Gilger BC, Mitra AK. Topical delivery of aqueous micellar resolvin E1 analog (RX-10045). Int J Pharm. 2016;498 (1-2):326-334. doi:10.1016/j.ijpharm.2015.12.037
63. Gjorstrup P, Pflugfelder SP, Pangelinan S, De Paiva CS. Resolvins protect against goblet cell loss and reduce corneal epithelial barrier disruption in a murine model of KCS. Invest Ophthalmol Vis Sci. 2008;122.

64. Li N, He J, Gjorstrup P, Bazan H. The resolvin E1 analogs, RX-10065 and RX-10005 improve tear production and decrease inflammation in a mouse dry eye model. Invest Ophthalmol Vis Sci. 2008;49:121.

65. Zhang F, Yang H, Pan Z, et al. Dependence of resolvin-induced increases in corneal epithelial cell migration on EGF receptor transactivation. Invest Ophthalmol Vis Sci. 2010;51 (11):5601-5609. doi:10.1167/iovs.09-4468

66. Downie LE, Ng SM, Lindsley KB, Akpek EK. Omega-3 and omega-6 polyunsaturated fatty acids for dry eye disease. Cochrane Database Syst Rev. 2019;2019(12). doi:10.1002/ 14651858.CD011016.pub2

67. Schmidt TA, Sullivan DA, Knop E, et al. Transcription, translation, and function of lubricin, a boundary lubricant, at the ocular surface. JAMA Ophthalmol. 2013;131(6):766-776. doi:10.1001/ jamaophthalmol.2013.2385

68. Lambiase A, Sullivan BD, Schmidt TA, et al. A two-week, randomized, double-masked study to evaluate safety and efficacy of lubricin $(150 \mu \mathrm{g} / \mathrm{mL})$ eye drops versus sodium hyaluronate (HA) $0.18 \%$ eye drops $\left(\right.$ Vismed $\left.^{(\mathbb{B}}\right)$ in patients with moderate dry eye disease. Ocul Surf. 2017;15(1):77-87. doi:10.1016/j. jtos.2016.08.004

69. Hou LF, He SJ, Li X, et al. Oral administration of artemisinin analog SM934 ameliorates lupus syndromes in MRL/lpr mice by inhibiting Th1 and Th17 cell responses. Arthritis Rheum. 2011;63 (8):2445-2455. doi:10.1002/art.30392

70. Yan YX, Shao MJ, Qi Q, et al. Artemisinin analogue SM934 ameliorates DSS-induced mouse ulcerative colitis via suppressing neutrophils and macrophages. Acta Pharmacol Sin. 2018;39 (10):1633-1644. doi:10.1038/aps.2017.185

71. Hou LF, He SJ, Li X, et al. Sm934 treated lupus-prone $\mathrm{NZB} \times \mathrm{NZW} F \mathrm{~F}$ mice by enhancing macrophage interleukin-10 production and suppressing pathogenic T cell development. PLoS One. 2012;7(2):e32424. doi:10.1371/journal.pone.0032424

72. Yang FM, Fan D, Yang XQ, et al. The artemisinin analog SM934 alleviates dry eye disease in rodent models by regulating TLR4/ NF-кB/NLRP3 signaling. Acta Pharmacol Sin. 2020. doi:10.1038/s41401-020-0484-5

73. Skulachev VP. Cationic antioxidants as a powerful tool against mitochondrial oxidative stress. Biochem Biophys Res Commun. 2013;441(2):275-279. doi:10.1016/j.bbrc.2013.10.063

74. Wei Y, Troger A, Spahiu V, et al. The role of SKQ1 (Visomitin) in inflammation and wound healing of the ocular surface. Ophthalmol Ther. 2019;8(1):63-73. doi:10.1007/s40123-0180158-2

75. Zernii EY, Gancharova OS, Baksheeva VE, et al. Mitochondriatargeted antioxidant SkQ1 prevents anesthesia-induced dry eye syndrome. Oxid Med Cell Longev. 2017;2017:1-17. doi:10.1155/ 2017/9281519

76. Voronkova YG, Popova TN, Agarkov AA, Zinovkin RA. Effect of SkQ1 on activity of the glutathione system and NADPH-generating enzymes in an experimental model of hyperglycemia. Biochem. 2015;80(12):1614-1621. doi:10.1134/ S000629791512010X

77. Brzheskiy VV, Efimova EL, Vorontsova TN, et al. Results of a multicenter, randomized, double-masked, placebo-controlled clinical study of the efficacy and safety of visomitin eye drops in patients with dry eye syndrome. Adv Ther. 2015;32 (12):1263-1279. doi:10.1007/s12325-015-0273-6

78. Gutierrez LS, Lopez-Dee Z, Pidcock K. Thrombospondin-1: multiple paths to inflammation. Mediators Inflamm. 2011;2011. doi: $10.1155 / 2011 / 296069$. 
79. Wight TN, Raugi GJ, Mumby SM, Bornstein P. Light microscopic immunolocation of thrombospondin in human tissues. $J$ Histochem Cytochem. 1985;33(4):295-302. doi:10.1177/ 33.4.3884704

80. Grimbert P, Bouguermouh S, Baba N, et al. Thrombospondin/ CD47 interaction: a pathway to generate regulatory $T$ cells from human CD4 + CD25 - T cells in response to inflammation. $J \quad$ Immunol. 2006;177(6):3534-3541. doi:10.4049/ jimmunol.177.6.3534

81. Doyen V, Rubio M, Braun D, et al. Thrombospondin 1 is an autocrine negative regulator of human dendritic cell activation. J Exp Med. 2003;198(8):1277-1283. doi:10.1084/jem.20030705

82. Jiménez B, Volpert OV, Crawford SE, Febbraio M, Silverstein RL, Bouck N. Signals leading to apoptosis-dependent inhibition of neovascularization by thrombospondin-1. Nat Med. 2000;6(1):41-48. doi:10.1038/ 71517

83. Saban DR, Bock F, Chauhan SK, Masli S, Dana R. Thrombospondin-1 derived from APCs regulates their capacity for allosensitization. $J$ Immunol. 2010;185(8):4691-4697. doi:10.4049/jimmunol.1001133

84. Sekiyama E, Nakamura T, Cooper LJ, et al. Unique distribution of thrombospondin-1 in human ocular surface epithelium. Invest Ophthalmol Vis Sci. 2006;47(4):1352-1358. doi:10.1167/ iovs.05-1305

85. Anshoo C, Paul H, Hart Charles A, Kaye Stephen B, Mark B, Ian G. Suppression of thrombospondin 1 and 2 production by herpes simplex virus 1 infection in cultured keratocytes PubMed. Mol Vis. 2005;11:163-168.

86. Scheef EA, Huang Q, Wang S, Sorenson CM, Sheibani N Isolation and characterization of corneal endothelial cells from wild type and thrombospondin-1 deficient mice. Mol Vis. 2007;13:1483-1495.

87. Turpie B, Yoshimura T, Gulati A, Rios JD, Dartt DA, Masli S. Sjögren's syndrome-like ocular surface disease in thrombospondin-1 deficient mice. Am J Pathol. 2009;175(3):1136-1147. doi:10.2353/ajpath.2009.081058

88. Tan X, Chen Y, Foulsham W, et al. The immunoregulatory role of corneal epithelium-derived thrombospondin-1 in dry eye disease. Ocul Surf. 2018;16(4):470-477. doi:10.1016/j.jtos.2018.07.005

89. Ruiz-Ederra J, Levin MH, Verkman AS. In situ fluorescence measurement of tear film $\left[\mathrm{Na}^{+}\right],[\mathrm{K}+],[\mathrm{Cl}-]$, and $\mathrm{pH}$ in mice shows marked hypertonicity in aquaporin-5 deficiency. Invest Ophthalmol Vis Sci. 2009;50(5):2132-2138. doi:10.1167/ iovs.08-3033

90. Levin MH, Jung KK, Hu J, Verkman AS. Potential difference measurements of ocular surface $\mathrm{Na}+$ absorption analyzed using an electrokinetic model. Invest Ophthalmol Vis Sci. 2006;47 (1):306-316. doi:10.1167/iovs.05-1082

91. Levin MH, Verkman AS. CFTR-regulated chloride transport at the ocular surface in living mice measured by potential differences. Invest Ophthalmol Vis Sci. 2005;46(4):1428-1434. doi:10.1167/iovs.04-1314

92. Hara S, Hazama A, Miyake M, et al. The effect of topical amiloride eye drops on tear quantity in rabbits - PubMed. Mol Vis. 2010;16:2279-2285.

93. Mirshahi M, Nicolas C, Mirshahi S, Golestaneh N, D’Hermies F, Agarwal MK. Immunochemical analysis of the sodium channel in rodent and human eye. Exp Eye Res. 1999;69(1):21-32. doi:10.1006/exer.1999.0675

94. Thelin WR, Johnson MR, Hirsh AJ, Kublin CL, Zoukhri D. Effect of topically applied epithelial sodium channel inhibitors on tear production in normal mice and in mice with induced aqueous tear deficiency. J Ocul Pharmacol Ther. 2012;28 (4):433-438. doi:10.1089/jop.2011.0157
95. Boyer J, Johnson MR, Ansede J, Donn K, Boucher R, Thelin W. $\mathrm{P}-321$, a novel long-acting epithelial sodium channel (ENaC) blocker for the treatment of dry eye disease. Invest Ophthalmol Vis Sci. 2013;54(15):957.

96. Yang JM, Wei ET, Kim SJ, Yoon KC. TRPM8 channels and dry eye. Pharmaceuticals. 2018;11(4):125. doi:10.3390/ph11040125

97. González-Muñiz R, Bonache MA, Martín-Escura C, GómezMonterrey I. Recent progress in TRPM8 modulation: an update. Int J Mol Sci. 2019;20(11):2618. doi:10.3390/ijms20112618

98. Bharate SS, Bharate SB. Modulation of thermoreceptor TRPM8 by cooling compounds. ACS Chem Neurosci. 2012;3(4):248-267. doi:10.1021/cn300006u

99. Sherkheli MA, Vogt-Eisele AK, Bura D, Beltrán Márques LR, Gisselmann G, Hatt H. Characterization of selective trpm8 1 igands and their structure activity response (S.A.R) relationship. $J$ Pharm Pharm Sci. 2010;13(2):242-253. doi:10.18433/j3n $88 \mathrm{n}$

100. Parra A, Madrid R, Echevarria D, et al. Ocular surface wetness is regulated by TRPM8-dependent cold thermoreceptors of the cornea. Nat Med. 2010;16(12):1396-1399. doi:10.1038/nm.2264

101. Chen G-L, Lei M, Zhou L-P, Zeng B, Zou F, Xu S-Z. Borneol is a TRPM8 agonist that increases ocular surface wetness. PLoS One. 2016;11(7):e0158868. doi:10.1371/journal.pone.0158868

102. Yang JM, Li F, Liu Q, et al. A novel TRPM8 agonist relieves dry eye discomfort. BMC Ophthalmol. 2017;17(1). doi:10.1186/ s12886-017-0495-2

103. Yoon HJ, Kim J, Yang JM, Wei ET, Kim SJ, Yoon KC. Topical TRPM8 agonist for relieving neuropathic ocular pain in patients with dry eye: a pilot study. J Clin Med. 2021;10(2):250. doi: $10.3390 / \mathrm{jcm} 10020250$

104. Yin Y, Le SC, Hsu AL, Borgnia MJ, Yang H, Lee SY. Structural basis of cooling agent and lipid sensing by the cold-activated TRPM8 channel. Science. 2019;363(6430):eaav9334. doi:10.1126/science.aav9334

105. Rodríguez-Arévalo S, Pujol E, Abás S, Galdeano C, Escolano C, Vázquez S. Synthesis, characterization and HPLC analysis of the (1S,2S,5R)-diastereomer and the enantiomer of the clinical candidate AR-15512. Molecules. 2021;26(4):906. doi:10.3390/ molecules 26040906

106. Moreno-Montañés J, Bleau AM, Jimenez AI. Tivanisiran, a novel siRNA for the treatment of dry eye disease. Expert Opin Investig Drugs. 2018;27(4):421-426. doi:10.1080/13543784.20 18.1457647

107. Bourinet E, Altier C, Hildebrand ME, Trang T, Salter MW, Zamponi GW. Calcium-permeable ion channels in pain signaling. Physiol Rev. 2014;94(1):81-140. doi:10.1152/ physrev.00023.2013

108. Gonzalez G, Garcia de la Rubia P, Gallar J, Belmonte C. Reduction of capsaicin-induced ocular pain and neurogenic inflammation by calcium antagonists. Invest Ophthalmol Vis Sci. 1993;34(12):3329-3335.

109. Benitez-Del-Castillo JM, Moreno-Montañés J, Jiménez-Alfaro I, et al. Safety and efficacy clinical trials for SYL1001, a novel short interfering RNA for the treatment of dry eye disease. Invest Ophthalmol Vis Sci. 2016;57(14):6447-6454. doi:10.1167/ iovs. 16-20303

110. Giannaccare G, Carnevali A, Senni C, Logozzo L, Scorcia V. Umbilical cord blood and serum for the treatment of ocular diseases: a comprehensive review. Ophthalmol Ther. 2020;9 (2):235-248. doi:10.1007/s40123-020-00239-9

111. Soni NG, Jeng BH. Blood-derived topical therapy for ocular surface diseases. $\mathrm{Br} \quad J$ Ophthalmol. 2016;100(1):22-27. doi:10.1136/bjophthalmol-2015-306842

112. Uccelli A, Moretta L, Pistoia V. Mesenchymal stem cells in health and disease. Nat Rev Immunol. 2008;8(9):726-736. doi:10.1038/ nri2395 
113. Lu X, Li N, Zhao L, et al. Human umbilical cord mesenchymal stem cells alleviate ongoing autoimmune dacryoadenitis in rabbits via polarizing macrophages into an anti-inflammatory phenotype. Exp Eye Res. 2020;191:107905. doi:10.1016/j.exer.2019.107905

114. Lee MJ, Ko AY, Ko JH, et al. Mesenchymal stem/stromal cells protect the ocular surface by suppressing inflammation in an experimental dry eye. Mol Ther. 2015;23(1):139-146. doi:10.1038/mt.2014.159
115. Weng $\mathrm{J}$, He C, Lai $\mathrm{P}$, et al. Mesenchymal stromal cells treatment attenuates dry eye in patients with chronic graft-versus-host disease. Mol Ther. 2012;20(12):2347-2354. doi:10.1038/mt.2012.208

116. Sáles CS, Johnston LJ, Ta CN. Long-term clinical course of dry eye in patients with chronic graft-versus-host disease referred for eye examination. Cornea. 2011;30(2):143-149. doi:10.1097/ ICO.0b013e3181e9b3bf

\section{Publish your work in this journal}

The Journal of Experimental Pharmacology is an international, peerreviewed, open access journal publishing original research, reports, reviews and commentaries on all areas of laboratory and experimental pharmacology. The manuscript management system is completely online and includes a very quick and fair peer-review system. Visit http://www.dovepress.com/testimonials.php to read real quotes from published authors. 